\title{
Elevated EN1 expression distinguishes basal-like human breast cancer.
}

Shahan Mamoor, MS ${ }^{1}$

$3 \quad$ shahanmamoor@gmail.com

East Islip, NY 11730

Patients diagnosed with basal-like breast cancer face a more aggressive disease course and more dismal prognosis than patients diagnosed with luminal A and luminal B breast cancer molecular subtypes (1-4). We mined published microarray data $(5,6)$ to understand in an unbiased fashion the most distinguishing transcriptional features of tumors from patients with basal or basal-like subtype breast cancer. We observed transcriptome-wide differential expression of engrailed homeobox 1, EN1, when comparing tumors of patients with basal-like breast cancer with that of other PAM50 molecular subtypes. EN1 mRNA was present at significantly higher quantities in the tumors of patients with basal-like breast cancer. Analysis of patient survival data revealed that EN1 primary tumor expression was correlated with distant metastasis-free survival in patients with breast cancer. Elevated EN1 expression appears to distinguish basal-like human breast cancer from the other molecular subtypes.

Keywords: basal subtype, basal-like breast cancer, EN1, systems biology of breast cancer, targeted therapeutics in breast cancer, molecular subtype. 
Classification of disease types and disease subtypes enables development of enhanced diagnostic capabilities, prognostic information, as well as an understanding of human disease that can support superior medical, surgical and radiological treatment of complex diseases such as cancer. Molecular subtypes in human breast cancer include luminal A, luminal B, basal or basal-like, and HER2+. Systems-level analyses, such as comparative tumor transcriptome analyses, can inform such approaches. We mined published microarray data $(5,6)$ to identify the most significant transcriptional differences when comparing human breast cancer molecular subtypes. Integrative microarray dataset analysis has provided data illuminating genes whose expression define basal-like subtype breast cancers, and we present evidence here.

\section{Methods}

We utilized datasets GSE74667 (5) and GSE158309 (6) for this global differential gene expression analysis of basal subtype human breast cancer in conjunction with GEO2R. GSE74667 was generated using Agilent-014850 Whole Human Genome Microarray 4x44K G4112F technology with $n=23$ basal-like breast tumors and $n=72$ breast tumors of other molecular subtypes (luminal A, luminal B, HER2-enriched, and normal-like); analysis was performed using platform GPL6480. GSE158308 was generated using Affymetrix Human Genome U133A Array technology with $n=52$ tumors from patients with basal-like breast breast and $n=410$ tumors from patients with luminal A, luminal B and HER2+ molecular subtypes; analysis was performed using platform GPL96. The Benjamini and Hochberg method of $p$-value adjustment was used for ranking of differential expression but raw $p$-values were used to assess statistical significance of global differential expression. Log-transformation of data was auto-detected, and the NCBI generated category of platform annotation was used. A statistical test was performed to evaluate whether EN1 gene expression was significantly different between the tumors of patients with basal and non-basal molecular subtype breast cancer using a two-tailed t-test with Welch's correction (PRISM 9.1.1 (225)). For Kaplan-Meier survival analysis, we used the Kaplan-Meier plotter tool (8) for correlation of EN1 mRNA expression levels with distant metastasis-free survival in $n=2765$ breast cancer patients, $n=630$ basal subtype breast cancer patients, $n=998$ luminal A subtype breast cancer patients, $n=673$ luminal B subtype breast cancer patients, $n=401$ HER2 + subtype breast cancer patients, and $n=63$ normal-like subtype breast cancer patients.

\section{Results}

EN1 is among the genes whose expression is most different when comparing basal-like breast cancers with breast cancers of other molecular subtypes.

We used published microarray data (5) to compare the whole tumor transcriptomes of 23 patients with basal subtype breast cancer and 72 patients with breast cancer of other molecular subtypes (luminal A, luminal B, HER2+ and normal-like) to identify genes whose expression marked the basal-like molecular subtype. We observed transcriptome-wide differential expression of engrailed homeobox 1, EN1, when comparing the tumors of patients with basal-like breast cancer with that of patients with luminal A, luminal B, HER2+ and normal-like subtype breast cancers (Table 1). When sorting each of the transcripts whose expression was measured globally, EN1 expression ranked 78 out of 31157 total transcripts, equating to $99.7 \%$ differential expression; this was statistically significant ( $p$-value: $3.41 \mathrm{E}-11$ ).

Analysis of a second published microarray dataset, comparing tumor transcriptome data from a separate cohort of patients (52 patients with basal-like breast cancer and 410 patients with breast cancer of other molecular subtype (luminal A, luminal B, and HER2+) (5) revealed transcriptome-wide differential expression of EN1 in patients with basal subtype human breast cancer (Table 2). When sorting each of the transcripts whose expression was measured globally, EN1 expression ranked 1437 out of 22283 total 
 \\ transcripts, equating to $93.6 \%$ differential expression; this was statistically significant ( $p$-value: $5.71 \mathrm{E}-04$ ). Thus, the data suggested differential expression of EN1 was a fundamental feature of basal-like breast cancer in humans rather than an artifact of a single microarray dataset or research group, or a finding derived from cell culture or an animal model that was not relevant to human breast cancer. \\ EN1 expression is significantly higher in basal-like breast cancers. \\ We obtained exact EN1 mRNA expression data for basal-like breast tumors and tumors from other molecular subtypes (luminal A, luminal B, HER2+ and normal-like) to determine magnitude and direction of EN1 gene expression change in basal-like breast cancer. EN1 was expressed at higher levels in the tumors of patients with basal-like breast cancer as compared to the tumors of patients from other PAM50 molecular subtypes, and this difference was statistically significant (Figure $1 ; p=0.0001$ ). EN1 was expressed at $2.476 \pm 2.131$ arbitrary units (A.U.) in basal-like breast cancer and $0.382 \pm 0.685$ A.U. in all other molecular subtypes (Figure 1). \\ EN1 expression correlates with distant metastasis-free survival in human breast cancer.}

We asked whether primary tumor expression of EN1 was correlated with survival outcomes in patients with basal-like breast cancer. We observed a correlation between primary tumor expression of EN1 and distant metastasis-free survival (DMFS) in patients with breast cancer which was statistically significant (Figure 2; $p$-value: 0.00015; hazard ratio: 1.35 (1.15-1.57)). EN1 primary tumor expression was a negative prognostic indicator in patients with breast cancer. Patients with low tumor expression of EN1 possessed median DMFS of 236.22 months, while patients with high tumor expression of EN1 possessed median DMFS of 222.81 months (Table 3).

EN1 primary tumor expression was not correlated with distant metastasis-free survival in basal subtype patients (Figure 2; $p$-value: 0.77; hazard ratio: 0.96 (0.71-1.28)), in luminal A subtype breast cancer (Figure 2; $p$-value: 0.39; hazard ratio: 0.87 (0.63-1.2)), in luminal B subtype breast cancer (Figure 2; $p$-value: 0.13; hazard ratio: 1.26 (0.94-1.68), in HER2+ breast cancer (Figure 2; $p$-value: 0.14; hazard ratio: $1.31(0.92-1.86)$ ), or in normal-like breast cancer (Figure 2; $p$-value: 0.33 ; hazard ratio: 1.61 (0.61-4.24)).

\section{Discussion}

We find here that EN1 is among the genes whose expression is most different when comparing basal-like breast cancers (BLBC) to cancers of other molecular subtypes, with significantly more EN1 mRNA in BLBC. Kaplan-Meier analysis of patient survival data revealed that EN1 expression was correlated with distant metastasis-free survival in patients with breast cancer, with higher expression of EN1 associated with inferior outcomes. EN1 may be one factor that specifies tumor subtype identity in basal-like breast cancer. 


\section{References}

1. Rakha, E.A., Reis-Filho, J.S. and Ellis, I.O., 2008. Basal-like breast cancer: a critical review. Journal of clinical oncology, 26(15), pp.2568-2581.

2. Milioli, H.H., Tishchenko, I., Riveros, C., Berretta, R. and Moscato, P., 2017. Basal-like breast cancer: molecular profiles, clinical features and survival outcomes. BMC medical genomics, 10(1), pp.1-17.

3. Foulkes, W.D., Smith, I.E. and Reis-Filho, J.S., 2010. Triple-negative breast cancer. New England journal of medicine, 363(20), pp.1938-1948.

4. Dietze, E.C., Sistrunk, C., Miranda-Carboni, G., O'regan, R. and Seewaldt, V.L., 2015. Triple-negative breast cancer in African-American women: disparities versus biology. Nature Reviews Cancer, 15(4), pp.248-254.

5. She, Q.B., Gruvberger-Saal, S.K., Maurer, M., Chen, Y., Jumppanen, M., Su, T., Dendy, M., Lau, Y.K.I., Memeo, L., Horlings, H.M. and van de Vijver, M.J., 2016. Integrated molecular pathway analysis informs a synergistic combination therapy targeting PTEN/PI3K and EGFR pathways for basal-like breast cancer. BMC cancer, 16(1), pp.1-16.

6. Heimes, A.S., Härtner, F., Almstedt, K., Krajnak, S., Lebrecht, A., Battista, M.J., Edlund, K., Brenner, W., Hasenburg, A., Sahin, U. and Gehrmann, M., 2020. Prognostic significance of interferon- $\gamma$ and its signaling pathway in early breast cancer depends on the molecular subtypes. International Journal of Molecular Sciences, 21(19), p.7178

7. Györffy, B., Lanczky, A., Eklund, A.C., Denkert, C., Budczies, J., Li, Q. and Szallasi, Z., 2010. An online survival analysis tool to rapidly assess the effect of 22,277 genes on breast cancer prognosis using microarray data of 1,809 patients. Breast cancer research and treatment, 123(3), pp.725-731. 


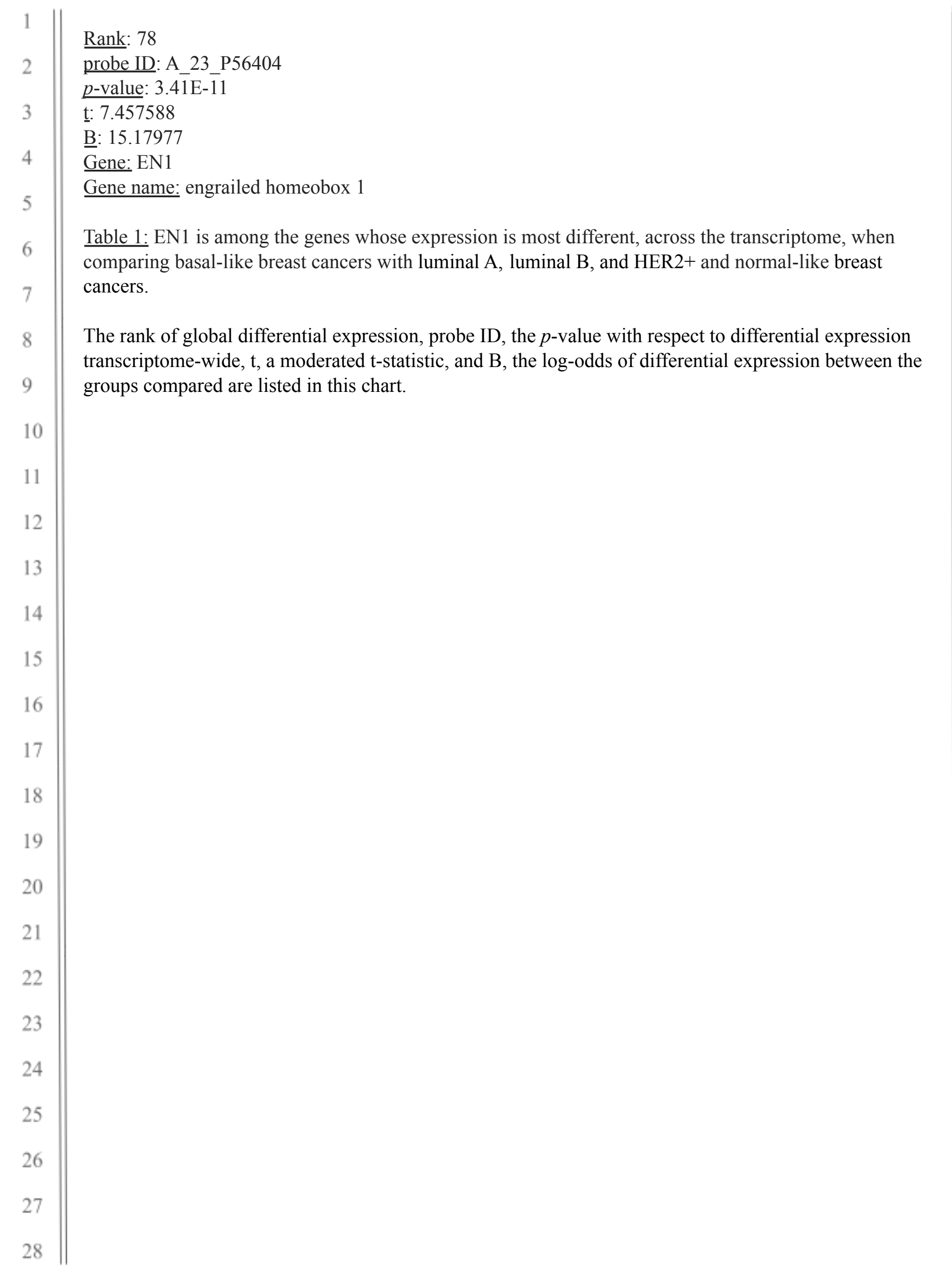




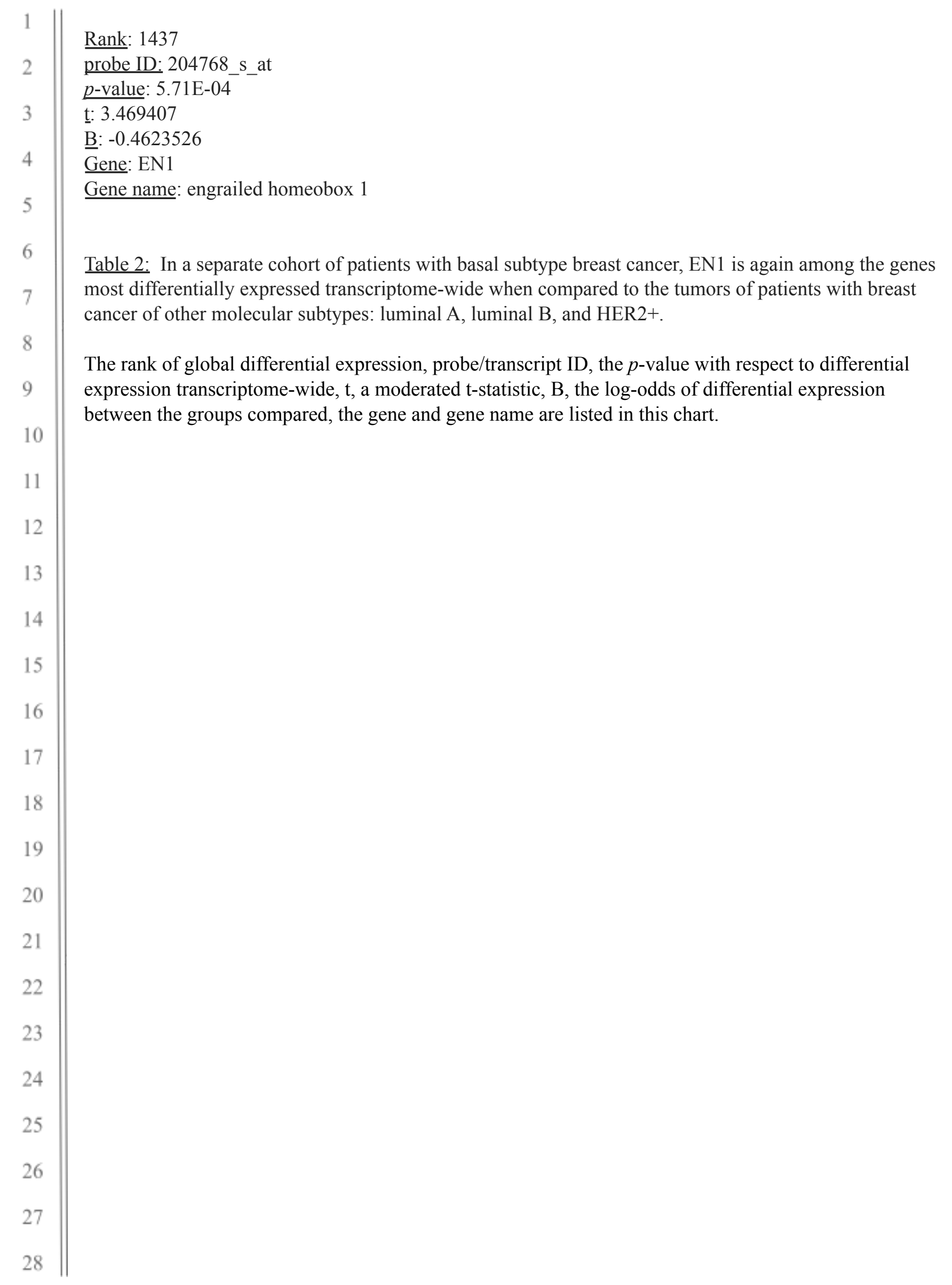




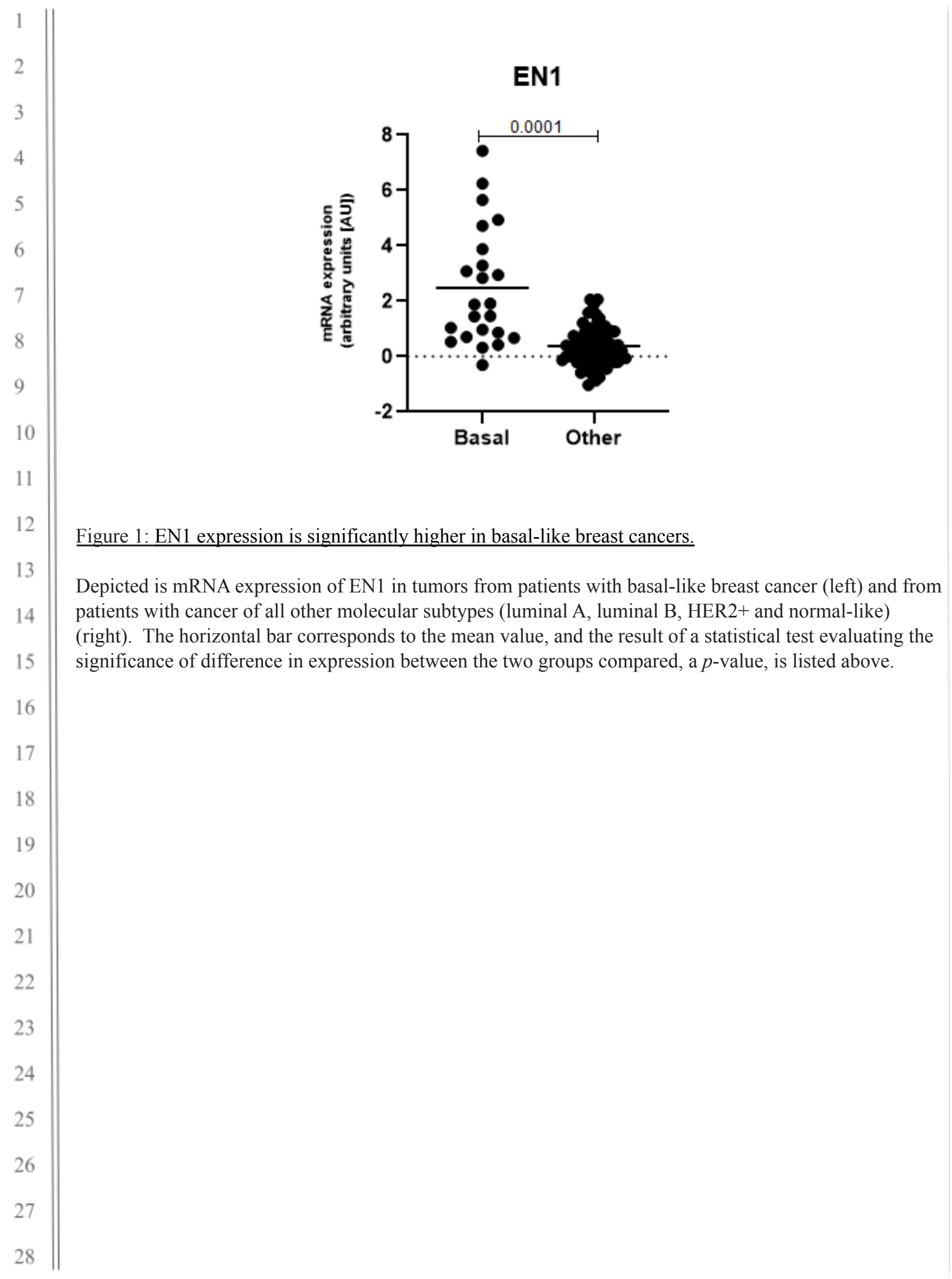




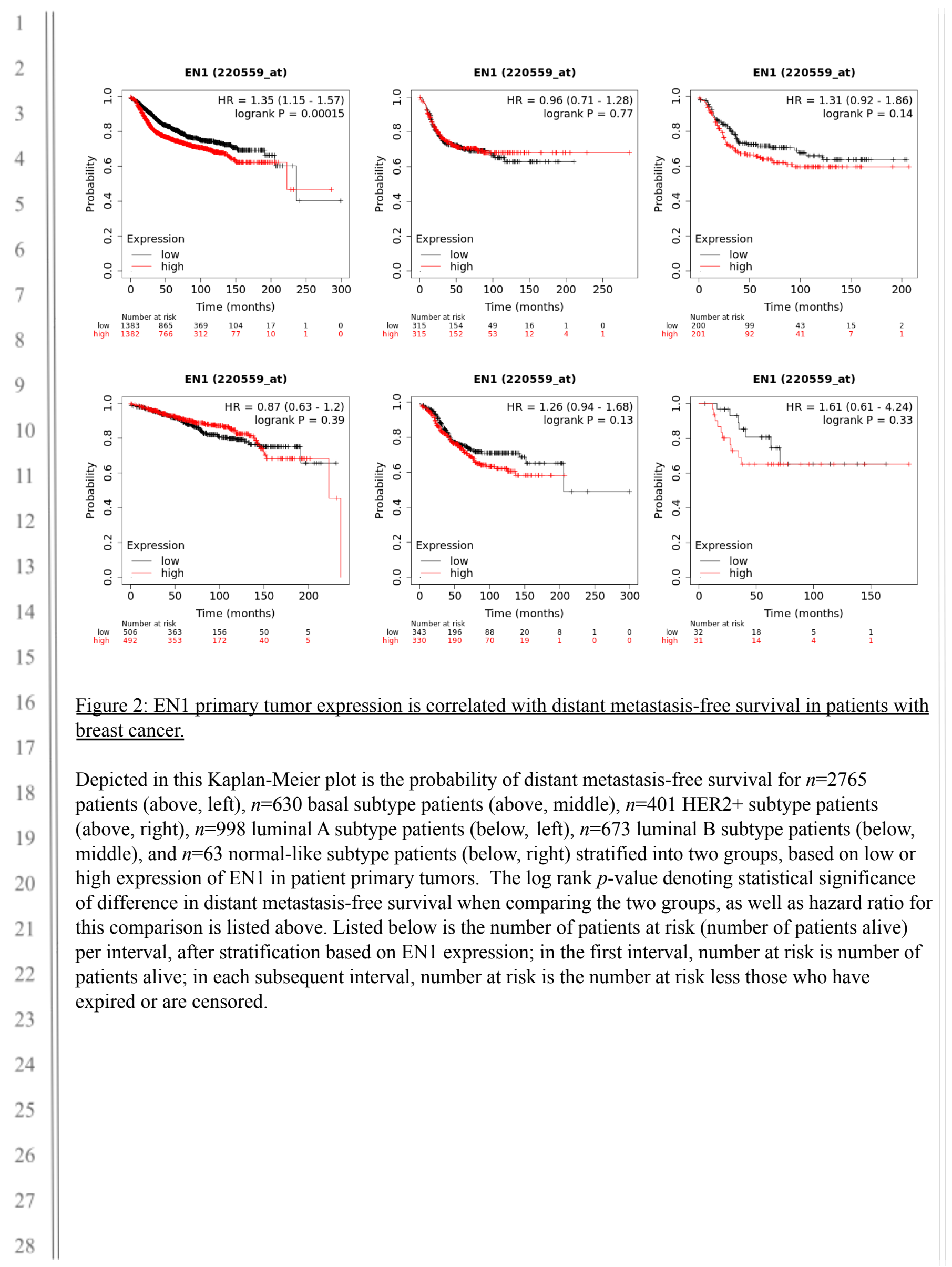




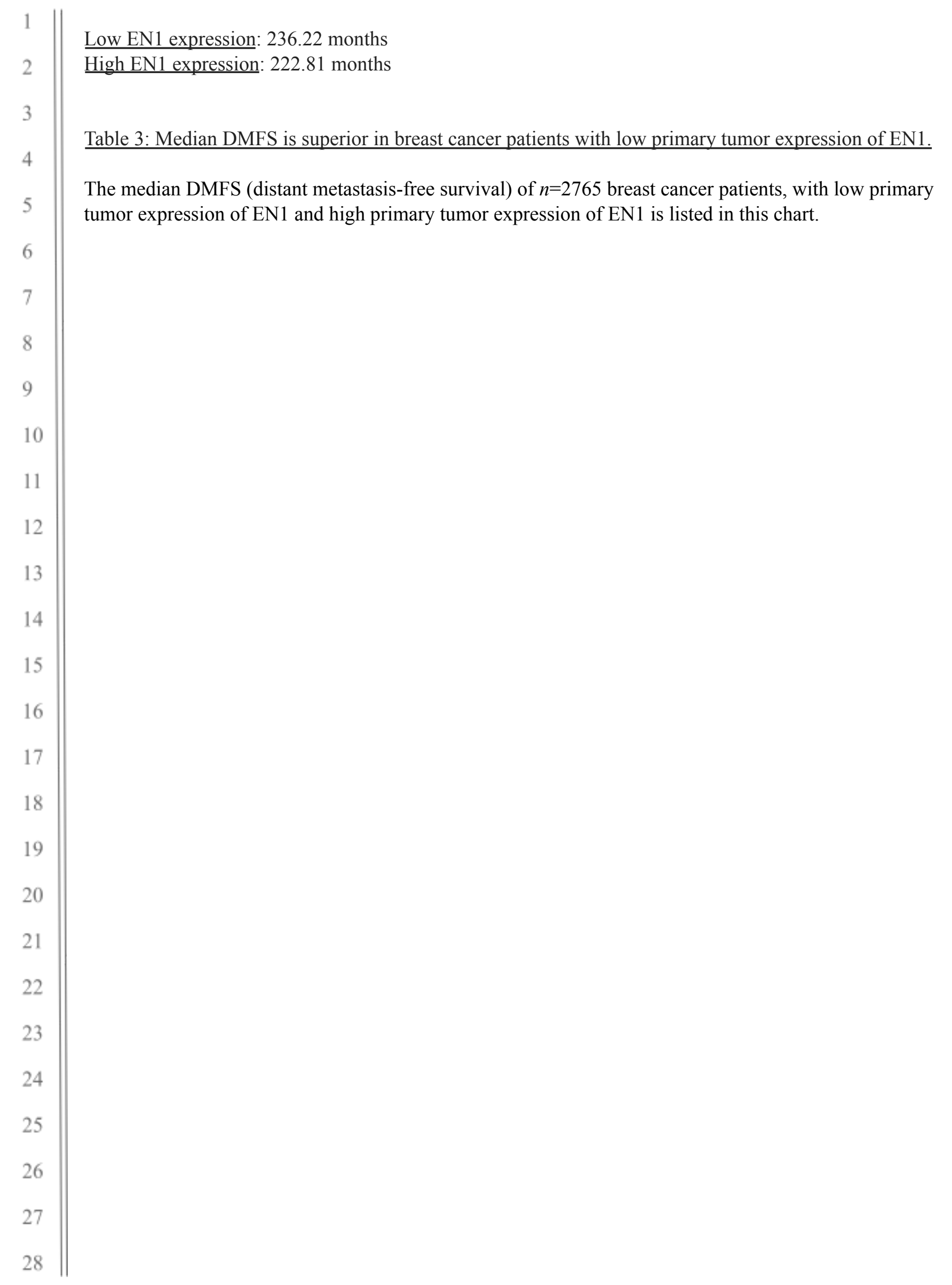

